IRSH 56 (20I I), p. 583 doi:10.1017/S002085901 100068X

(C) 20 I I Internationaal Instituut voor Sociale Geschiedenis

\title{
NOTES ON CONTRIBUTORS
}

Gabriel Aladrén, Fluminense Federal University, Campus do Gragoatá, Prof. Marcos Rua Waldemar de Freitas Reis, Bloco O, Room 505, 242 10-380 Niterói, RJ, Brazil; e-mail: gabrielaladren@yahoo.com.br

Hans de Beer, Internationaal Instituut voor Sociale Geschiedenis, Cruquiusweg 3 I, Ior9 AT Amsterdam, The Netherlands; e-mail: hdb@iisg.nl Sidney Chalboub, Department of History, Universidade Estadual de Campinas (UNICAMP), Rua Cora Coralina, s/n, Campinas SP I 3083-896, Brazil; e-mail: chalhoub@unicamp.br

William Gervase Clarence-Smith, School of Oriental and African Studies, University of London, Thornhaugh Street, Russell Square, London WCI $\mathrm{H} \circ \mathrm{XG}$, United Kingdom; e-mail: wc2@soas.ac.uk

Rena Fuks-Mansfeld, Internationaal Instituut voor Sociale Geschiedenis, Cruquiusweg 3 I, I0I9 AT Amsterdam, The Netherlands; e-mail: rfu@iisg.nl Sharif Gemie, School of Humanities and Social Sciences, University of Glamorgan, Pontypridd CF 37 IDL, United Kingdom; e-mail: sgemie@glam.ac.uk Steven King, School of Historical Studies, University of Leicester, University Road, Leicester, LE I 7 RH, United Kingdom; e-mail: sak28@leicester.ac.uk Ad Knotter, Sociaal Historisch Centrum voor Limburg/Maastricht University, St Pieterstraat 7, 62 I I JM Maastricht, The Netherlands; e-mail: a.knotter@maastrichtuniversity.nl

Pablo Sánchez León, Departamento de Derecho Constitucional e Historia del Pensamiento y de los Movimientos Sociales y Políticos, Universidad del País Vasco, Campus de Leioa, Bilbao 48940, Spain; e-mail: psleon@gmail.com Louise Rees, School of Humanities and Social Sciences, University of Glamorgan, Pontypridd $\mathrm{CF}_{37}$ IDL, United Kingdom; e-mail: louise8ringram@gmail.com

Marina de Regt, Internationaal Instituut voor Sociale Geschiedenis, Cruquiusweg 3 I, I0I9 AT Amsterdam, The Netherlands; e-mail: mre@iisg.nl 